\title{
Fibrinolytic Effect of Tissue Plasminogen Activator on Cerebral Embolism in Stroke-Prone Spontaneously Hypertensive Rats
}

\author{
Hideki Kimata, ${ }^{*, a}$ Katsuaki Nakajima, ${ }^{a}$ Hideo Suzuki, ${ }^{a}$ Takashi KoIDe, ${ }^{a}$ Susumu Yamamoto ${ }^{a}$ and Tsutomu Narita ${ }^{b}$ \\ Tokyo Research Laboratories, Kowa Co., Ltd., Noguchi-cho, Higashimurayama, Tokyo 189, Japan and Asahi Chemical Industry Co., Ltd., ${ }^{b}$ Nobeoka, \\ Miyazaki 882, Japan. Received May 10, 1991
}

To study the thrombolytic effect of tissue plasminogen activator (t-PA) on cerebral emboli, we characterized cerebral embolization in stroke-prone spontaneously hypertensive rats (SHRSPs) and Wistar Kyoto rats (WKYs). $\left[{ }^{125} \mathrm{I}\right]$ Fibrin clot particles $(20-100 \mu \mathrm{m}$ diameter) were injected twice at an interval of $90 \mathrm{~min}$ into the left internal carotid artery of WKYs and SHRSPs. After each injection, spontaneous embolus dissolution was monitored with a $\gamma$-ray detector placed on the head of the embolic rats. Embolus dissolution was spontaneously generated in $15 \mathrm{~min}$ after the injection of fibrin clots. In WKYs, $21 \%$ and $42 \%$ of the clots were dissolved 30 and 90 min after the second embolization, respectively. On the other hand, the spontaneous embolus dissolution in SHRSPs was significantly lower than that of WKYs, indicating that the endogenous fibrinolytic ability of SHRSPs is less potent than that of normotensive rats. The intravenous administration of t-PA at doses of 75, 250 and $750 \mu \mathrm{g} / \mathrm{kg}$ caused a dose-dependent embolus dissolution in SHRSPs. Furthermore, systemically applied t-PA produced embolus dissolution without causing systemic plasminogen activation, fibrinogen breakdown or bleeding.

In conclusion, the intravenous administration of $t-P A$ produces selective embolus dissolution without systemic fibrino(geno)lysis in a cerebral embolic SHRSP.

Keywords cerebral embolism; stroke-prone spontaneously hypertensive rat; Wistar Kyoto rat; tissue plasminogen activator; fibrinolytic therapy

\section{Introduction}

Mammalian plasma contains an enzymatic system capable of dissolving the fibrin in blood clots. One component of this fibrinolytic system, the serine protease plasminogen activators, generates the active enzyme plasmin by limited proteolysis of the zymogen plasminogen. Plasmin subsequently dissolves the fibrin network of a clot to form soluble products. ${ }^{1)}$ Two major plasminogen activators are tissue plasminogen activator ( $t-P A)$, found in several tissue extracts, and urokinase plasminogen activator (u-PA) isolated from human urine. The t-PA has been extracted from a cultured human melanoma cell line $^{2)}$ and produced by recombinant deoxyribonucleic acid (DNA) techniques in bacterial cells ${ }^{3)}$ or mammalian cells. ${ }^{4)}$ In this study we used the t-PA obtained from cultured human diploid fibroblasts. ${ }^{\text {5) }}$

According to several studies in vitro, t-PA has a much higher affinity for fibrin compared with u-PA, and it increases plasminogen binding to a fibrin clot. ${ }^{6,7)}$ Therefore, it has been considered that the activation of plasminogen by fibrin-bound t-PA causes the efficient generation of plasmin on the thrombus without systemic plasminogen activation, which can produce indiscriminate digestion of coagulation proteins and significantly increase the risk of hemorrhage during treatment.

Atherothrombosis and thromboembolism are the main causes of acute stroke, and several thrombolytic agents such as u-PA and streptokinase (SK) have been routinely used for the therapy. However, there is criticism against the therapeutic use of thrombolytic agents because of the possibility of intracerebral hemorrhage during treatment. ${ }^{8)}$ Particularly, the intravenous infusion of large doses of u-PA or SK has generally been contraindicated to initiate the bleeding tendency because it can bring on an accerelation of systemic fibrino(geno)lysis. ${ }^{9)}$ On the other hand, if the early elimination of thrombi has not been achieved in acute stroke patients, permanent neurological deficits are difficult to avoid. Recently, intraarterial infusion with low doses of
u-PA or SK has been used to demonstrate clinical improvement without intracerebral hemorrhagic complication. ${ }^{10,11)}$ Nevertheless, the intravenous application of a thrombolytic agent is clinically most desirable in order to avoid the burden of catheterization in patients and to practice rapid therapy. The t-PA is expected to possess "clot-selective" thrombolysis and may be systemically applicable for the therapy of acute stroke.

Previously, we reported that the intraarterial administration of t-PA produced embolus dissolution in an experimental cerebral embolic model of Wistar rats. ${ }^{12)}$ However, hypertension is one of the important systemic risk factors in the pathogenesis of stroke, and stroke-prone spontaneously hypertensive rat (SHRSP) is a good pathogenetic animal model for studies on stroke in humans as advocated by Yamori et al. ${ }^{13)}$ In the present study, we prepared a thrombo-embolic stroke model by injecting $\left[{ }^{125} \mathrm{I}\right]$ fibrin clot particles into the left internal carotid artery in SHRSPs and normotensive Wistar Kyoto rats (WKYs), and characterized the deterioration of defensive fibrinolysis in SHRSPs. Furthermore, we demonstrated that $\mathrm{t}$ PA dissolved cerebral emboli without causing systemic fibrino(geno)lysis in a cerebral embolic SHRSP.

\section{Materials and Methods}

Materials The t-PA (sigle chain form, molecular weight ca. 63000) was purified from a confluent culture of human diploid fibroblasts as previously described. ${ }^{\text {s) }}$ The enzymatic activity of t-PA was approximately $40000 \mathrm{U} / \mathrm{mg}$ protein, which formed the same lysis area with u-PA at a concentration of $10 \mathrm{IU} / \mathrm{ml}$ on a fibrin plate according to the method reported by Rijken et al. ${ }^{14)}$ Human fibrinogen (Fbg) was purchased from Sigma Co., Ltd., St. Louis. Tissue thromboplastin (Simplastin ${ }^{\otimes}$, Warner-Lambert Co., Ltd., New Jersey) was used for plasma clot formation. The t-PA was dissolved with saline containing $0.02 \%$ Tween -80 .

Iodination of Fbg Fbg was labelled with $\mathrm{Na}\left[{ }^{125} \mathrm{I}\right](17.4 \mathrm{Ci} / \mathrm{mg}, \mathrm{NEN}$ Products, Boston) using solid state lactoperoxidase and glucose oxidase (Enzymobeads ${ }^{\circledR}$, Bio-Rad, Richmond) according to the method reported by David and Reisfeld. ${ }^{15)}$ The $\left.{ }^{125} \mathrm{I}\right] \mathrm{Fbg}$ showed $0.5 \times 10^{8} \mathrm{cpm} / \mathrm{nmol}$ specific radioactivity and approximately $90 \%$ clottability, and the same pattern as that of the respective native $\mathrm{Fbg}$ on sodium dodecyl sulfate- 
polyacrylamide gel electrophoresis (SDS-PAGE).

Preparation of the Suspension with [ ${ }^{125}$ I]Fibrin Clot Particles Plasma of $(2 \mathrm{ml})$ obtained from a male Jla: Wistar rat was mixed with $200 \mu \mathrm{l}$ of $\left[{ }^{125} \mathrm{I}\right] \mathrm{Fbg}\left(4 \times 10^{7} \mathrm{cpm}\right)$ and $10 \mu \mathrm{l}$ of tissue thromboplastin $(11 \mathrm{mg} / \mathrm{ml})$ After incubation at $37^{\circ} \mathrm{C}$ for $20 \mathrm{~min}$, the formed plasma clot was washed 3 times with $0.175 \mathrm{M} \mathrm{NaCl}$ (saline) and then powdered in a mortar under a freezing condition with liquid nitrogen. The powdered $\left[{ }^{125} I\right]$ fibrin clots were further homogenized using a Teflon homogenizer after an addition of $5 \mathrm{ml}$ saline. The $\left[{ }^{125} \mathrm{I}\right]$ fibrin clot particles $(20-100 \mu \mathrm{m})$ had a specific activity of approximately $4 \times 10^{3} \mathrm{cpm} / \mu \mathrm{g}$ protein.

Preparation of Cerebral Embolic Model Male SHRSPs were maintained and breeded in our laboratories after transfer from the Department of Pathology, Kinki Medical University (Osaka, Japan). SHRSPs, weighing ca. $310 \mathrm{~g} \mathrm{(23-30} \mathrm{weeks} \mathrm{of} \mathrm{age),} \mathrm{and} \mathrm{Male} \mathrm{WKYs,} \mathrm{weighing} \mathrm{ca.} 400 \mathrm{~g}$ (23-30 weeks of age) were used. Tail systolic blood pressures measured prior to the experiments in both strains were $257 \pm 3 \mathrm{mmHg}$ (in SHRSPs, $n=23$ ) and $132 \pm 4 \mathrm{mmHg}$ ( in WKYs, $n=5$ ), respectively. Preparation for the embolic model was based upon the previous paper. ${ }^{12)}$ The rats were anesthetized with an intraperitoneal injection of urethane/ chloralose/water $(500 \mathrm{mg} / 40 \mathrm{mg} / \mathrm{ml}, 1.5 \mathrm{ml} / \mathrm{kg}$ body weight). After a polyethylene tracheostomy tube was inserted, the left carotid artery was exposed and bifurcations (occipital and pterygopalatine arteries) of the left internal carotid artery were ligated. A temporary clip was then placed at the origin of the external carotid artery. The external carotid artery was cannulated retrogradely with PE-10 (Intramedic ${ }^{\circledR}$ ) tubing, the clip removed and the tip was positioned near the origin of the internal carotid artery. Through this catheter, $50 \mathrm{U} / 50 \mu \mathrm{l}$ heparin and $50 \mu \mathrm{l}$ of $2 \%$ $\mathrm{NaI}$ were simultaneously injected. Then, the cerebral embolization was produced in the left hemisphere by an injection of $50 \mu \mathrm{l}\left[{ }^{125} \mathrm{I}\right]$ fibrin clot suspension $\left(3 \times 10^{4} \mathrm{cpm} / 7.5 \mu \mathrm{g}\right.$ protein) containing $1 \%$ bovine serum albumin (BSA) in saline via the same catheter.

Measurement of Cerebral Embolus Dissolution The suspension of $\left[{ }^{125}\right.$ I] fibrin clot particles was injected twice consecutively at an interval of $90 \mathrm{~min}$ through the external carotid artery of the animals. Disappearance of radioactivity in the brain following the embolus dissolution was measured with a $\gamma$-ray detector (Aloka TCS-153, Japan) which was stereotaxically placed on the head of a cerebral embolic rat. The changes in radioactivity were continuously recorded through out the experimental period. The quantity of embolus dissolution was shown as the percentage of the initial value obtained from the embolus dissolution curve.

Administration of t-PA. Thirty minutes after the second embolization in SHRSPs, $\mathrm{t}$-PA was administered with a bolus $(1 \mathrm{ml} / \mathrm{kg}$ for $20 \mathrm{~s})$ into the caudal vein. The embolus dissolution was continuously recorded until 60 min after administration. A control group was treated with the same volume of vehicle (saline containing $0.02 \%$ Tween-80). The t-PA groups were studied with three doses of 75,250 and $750 \mu \mathrm{g} / \mathrm{kg}$.

Levels of Plasminogen and Fbg in Plasma after t-PA Administration Blood samples were taken from the abdominal vena cava just after the end of the experiments on embolus dissolution. The blood samples ( 9 volumes) were collected in plastic tubes containing $3.8 \%$ trisodium citrate ( 1 volume) and centrifuged at $4^{\circ} \mathrm{C}$. Plasma was frozen and stored at $-70^{\circ} \mathrm{C}$ for assay. Plasminogen plasma levels were measured with the chromogenic substrate S-2251 after activation with a 2000 IU of $\mathrm{u}-\mathrm{PA} / 200 \mu \mathrm{l}$ euglobulin fraction of plasma at $37^{\circ} \mathrm{C}$ for $5 \mathrm{~min} .^{16)} \mathrm{Fbg}$ plasma levels were determined according to the method of Quick ${ }^{171}$ after the addition of trans-4-aminomethyl cyclohexane carboxylic acid ( $t$-AMCHA) to the plasma samples to prevent proteolysis.

\section{Results}

Spontaneous Embolus Dissolution in WKYs and SHRSPs $\left[{ }^{125}\right.$ I]Fibrin clot suspension was consecutively injected twice at an interval of $90 \mathrm{~min}$. The radioactivity measured with a $\gamma$-ray detector placed on the heads of the animals was accumulated by consecutive injection. The initial radioactivities in WKYs and SHRSPs were $6420 \pm 670$ (mean \pm S.E.) and $7200 \pm 560 \mathrm{cpm}$ at the first injection, and $10030 \pm 570$ and $11990 \pm 1560 \mathrm{cpm}$ at the second injection, respectively. These values between WKYs and SHRSPs were not significantly different. After the injection of fibrin clots to WKYs and SHRSPs, the radioactivity spontaneously decreased. As previously reported, ${ }^{12)}$ the sponta- neous decrease in radioactivity is the dissolution of fibrin clots produced by an endogenous fibrinolytic system. The time course of the spontaneous embolus dissolution in WKYs and SHRSPs was shown in Table I. The spontaneous dissolution after the second embolization was much smaller than that after the first embolization in both the WKYs and SHRSPs. Furthermore, SHRSPs showed the less potent dissolution when compared with WKYs. The dissolution in WKYs after the second embolization was $21 \%$ at $30 \mathrm{~min}$ and $42 \%$ at $90 \mathrm{~min}$. On the other hand, the dissolution in SHRSPs was $10 \%$ at $30 \mathrm{~min}$ and $26 \%$ at $90 \mathrm{~min}$. The values in SHRSP s were significantly different from those in WKYs.

Effect of t-PA on Cerebral Embolism in SHRSPs As mentioned above, the spontaneous dissolution after the second embolization was smaller than that after the first embolization. Therefore t-PA was expected to show more effective clotlysis after the second embolization than after the first. Thirty minutes after the second embolization in SHRSPs, t-PA and the vehicle were intravenously administered with a bolus. The typical dissolution curve of cerebral emboli in a t-PA-treated animal is shown in Fig. 1. The embolus dissolution was generated soon after the administration of t-PA. The dissolution of cerebral emboli was presented as a percentage of the initial value, which was radioactivity $30 \mathrm{~min}$ after the second injection of fibrin clots. The magnitudes of the embolus dissolution 30 and 60 min after t-PA administration are shown in Fig. 2. The $\mathrm{t}-\mathrm{PA}$ in doses of 75,250 and $750 \mu \mathrm{g} / \mathrm{kg}$ showed a dose-

TABLE I. Spontaneous Dissolution of Cerebral Emboli after the Repeated Injection of $\left[{ }^{125} \mathrm{I}\right]$ Fibrin Clots into the Internal Carotid Artery in WKYs and SHRSPs

\begin{tabular}{|c|c|c|c|c|c|c|}
\hline \multirow{3}{*}{ Animal } & \multicolumn{6}{|c|}{ Dissolution of emboli (\%) } \\
\hline & \multicolumn{3}{|c|}{ 1st embolization } & \multicolumn{3}{|c|}{ 2nd embolization } \\
\hline & $30 \mathrm{~min}$ & $60 \mathrm{~min}$ & $90 \mathrm{~min}$ & $30 \mathrm{~min}$ & $60 \mathrm{~min}$ & $90 \mathrm{~min}$ \\
\hline $\begin{array}{l}\text { WKY } \\
\text { SHRSP }\end{array}$ & $\begin{array}{l}30.6 \pm 0.7 \\
19.3 \pm 1.1^{a}\end{array}$ & $\begin{array}{l}50.6 \pm 1.2 \\
36.1 \pm 2.2^{a)}\end{array}$ & $\begin{array}{l}59.9 \pm 1.4 \\
44.5 \pm 2.5^{a)}\end{array}$ & $\begin{array}{l}20.5 \pm 0.3 \\
10.0 \pm 1.1\end{array}$ & $\begin{array}{l}34.6 \pm 1.1 \\
20.5 \pm 1.8^{a}\end{array}$ & $\begin{array}{l}41.9 \pm 1.0 \\
26.1 \pm 1.5^{a)}\end{array}$ \\
\hline
\end{tabular}

Values represent the mean + S.E. of 5 animals. a) $p<0.01$ (significant difference compared to the WKY group by unpaired Student's $t$-test). Initial radioactivities after the 1 st and 2 nd injection of $\left[{ }^{125} I\right]$ fibrin clots were mentioned in text.

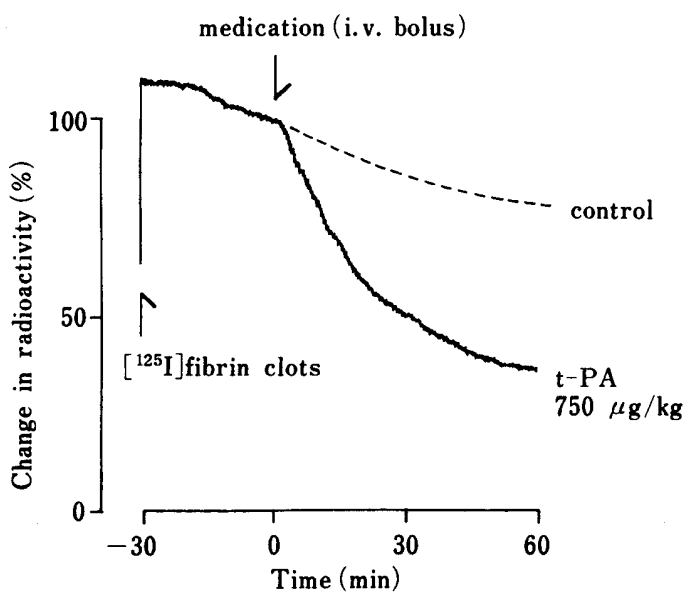

Fig. 1. Typical Dissolution Curve of Cerebral Emboli after Intravenous Administration of t-PA in SHRSPs

Decrease in radioactivity shows embolus dissolution. The radioactivity at $0 \mathrm{~min}$, namely, $30 \mathrm{~min}$ after the second injection of fibrin clots, was $8640 \mathrm{cpm}$ and represents $100 \%$. Broken line shows the predicted dissolution curve of control. 


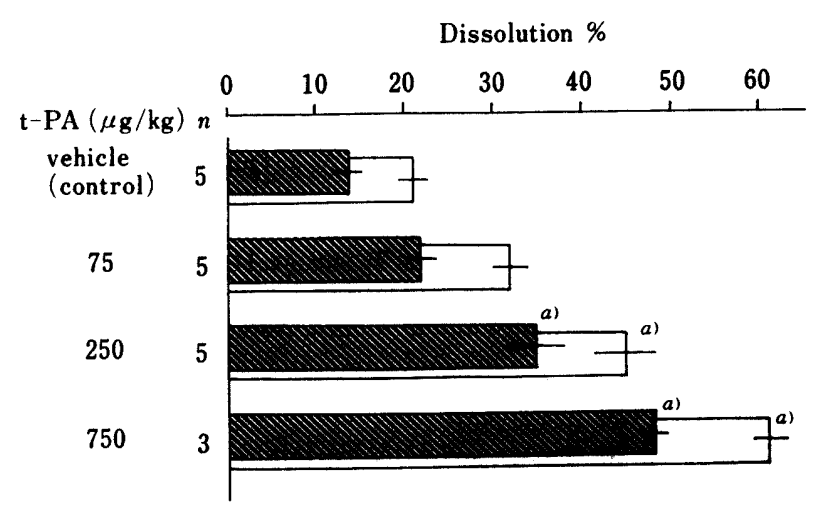

Fig. 2. Embolus Dissolution Effect of t-PA Intravenously Administered to Cerebral Embolic Model of SHRSPs

DV embolus dissolution 30 min after administration; $\square$ embolus dissolution $60 \mathrm{~min}$ after administration. Embolus dissolution (decrease in radioactivity) is represented as a percentage of initial radioactivity. Initial radioactivities $(30 \mathrm{~min}$ after the second injection of fibrin clots) in the control, 75, 250 and $750 \mu \mathrm{g} / \mathrm{kg} \mathrm{t}-\mathrm{PA}$ groups were $9950 \pm 610,9680 \pm 1180,8160 \pm 880$ and $9850 \pm 940 \mathrm{cpm}$, respectively. Column lengths represent the mean + S.E. a) $p<0.01$ (significant difference compared to the control group by Bartlett's test for comparison of variances and by Dunnett's method for evaluation).

TABLE II. Plasma Levels of Plasminogen and Fbg after Intravenous Administration of t-PA to Cerebral Embolic Model in SHRSPs

\begin{tabular}{|c|c|c|c|c|}
\hline \multirow[b]{2}{*}{ Drug } & \multirow[b]{2}{*}{ Dose } & \multirow[b]{2}{*}{$n$} & \multicolumn{2}{|c|}{ Plasma levels } \\
\hline & & & $\begin{array}{l}\text { Plasminogen } \\
(\mathrm{CU} / \mathrm{ml})\end{array}$ & $\underset{(\mathrm{mg} / \mathrm{dl})}{\mathrm{Fbg}}$ \\
\hline Control & & 5 & $3.36 \pm 0.04$ & $173 \pm 15$ \\
\hline t-PA & $75 \mu \mathrm{g} / \mathrm{kg}$ & 5 & $3.32 \pm 0.05$ & $161 \pm 4$ \\
\hline t-PA & $250 \mu \mathrm{g} / \mathrm{kg}$ & 5 & $3.44 \pm 0.04$ & $155 \pm 8$ \\
\hline t-PA & $750 \mu \mathrm{g} / \mathrm{kg}$ & 3 & $3.41 \pm 0.01$ & $150 \pm 9$ \\
\hline
\end{tabular}

Values represent the mean \pm S.E.

dependent embolus dissolution. Approximately $60 \%$ of fibrin clots were dissolved $60 \mathrm{~min}$ after the administration of $750 \mu \mathrm{g} / \mathrm{kg} \mathrm{t}-\mathrm{PA}$, while dissolution in the control group was less than $20 \%$.

Systemic administration of t-PA did not cause evident bleeding from the surgical site as compared to the control rats. Plasma samples were obtained at the end of experiments. As shown in Table II, the plasminogen and Fbg levels in plasma of the groups administered with t-PA were not different from the control group levels.

\section{Discussion}

Previously we reported that fibrin clots injected to the left hemicerebrum vasculature in Wistar rats were spontaneously dissolved by an endogenous fibrinolytic system since the embolus dissolution is blocked by a plasmin inhibitor, $t$-AMCHA. ${ }^{12)}$ In the present study, the spontaneous embolus dissolution was observed in WKYs as well as SHRSPs after the injection of fibrin clots. In the WKYs, 21 and $42 \%$ of clots were dissolved 30 and $90 \mathrm{~min}$ after the second embolization, respectively. These values were approximately the same as those previously obtained in Wistar rats. However, the spontaneous embolus dissolution produced in SHRSPs was significantly lower than that of WKYs. These results indicate that the defensive fibrinolytic ability in SHRSPs is deteriorated and suggest that an abnormality of the fibrinolytic system may relate to the pathogenesis of stroke in SHRSPs. Coyle et al. ${ }^{18)}$ have reported that the sudden occlusion of the middle cerebral artery in SHRSPs invariably results in a large infarction. They suggested that genetic factors may contribute to the difference in the susceptibility to infarction between SHRSPs and Wistar rats. Tagami et al. ${ }^{19)}$ also showed the occurrence of arterial occlusion with monocyte adhesion and fibrin deposition in cerebral perforating arteries after symptoms of stroke in SHRSPs. As hypertension is one of the important systemic risk factors in the pathogenesis of stroke, the SHRSP is a good pathogenetic animal model for studies on stroke in humans.

Thrombolytic therapy of stroke with t-PA is potentially more attractive than that with $u-P A$ or SK since t-PA causes clot-selective fibrinolysis through the activation of plasminogen. Although many investigators have reported the thrombolytic effects of t-PA in various animal models, ${ }^{20-27)}$ there are few studies on the direct proof of embolus dissolution with t-PA in the brain. ${ }^{28)}$ Previously we autoradiographically observed the presence of microthrombi in the hemicerebrum of Wistar rats after injection of $\left[{ }^{125} \mathrm{I}\right]$ fibrin clots. ${ }^{12)}$ Although several investigators have also used radiolabelled materials to study thrombolysis, ${ }^{29)}$ the methods they used were not sufficient to quantify cerebral embolism or thrombosis. In the present study we used a $\gamma$-ray detector placed stereotaxically on the head of the cerebral embolic rat after the injection of $\left[{ }^{125} I\right]$ fibrin clots, and we continuously monitored the radioactivity. This method allowed for the quantitative analysis of embolus dissolution with t-PA. As mentioned above, the endogenous fibrinolytic ability of SHRSPs is less potent than that of normotensive rats. Therefore it is of worth to study the thrombolytic effect of t-PA on cerebral embolism produced in SHRSPs. In the present study, the bolus intravenous injection of t-PA showed a dose-dependent embolus dissolution in SHRSPs. In addition, embolus dissolution was observed through the experiment periods of $60 \mathrm{~min}$. These results were noteworthy since the thrombolytic effect of t-PA was sustained beyound its time of clearance from circulation. The half life-time of t-PA in circulation is reportedly $1-2 \mathrm{~min}$ in rats. ${ }^{30)} \mathrm{A}$ discrepancy between the effect and blood levels of t-PA has been observed in a jugular vein thrombosis model of rabbits. ${ }^{31)}$ We previously reported that t-PA is more preferably accumulated to thrombi than u-PA in thrombin-induced DIC (disseminated intravascular coagulation) rats. ${ }^{32,33)}$ The sustained thrombolytic effect of t-PA after the bolus injection is possibly due to the localization of t-PA on thrombi. The present study suggests that the bolus injection of t-PA could be effective for thrombosis therapy.

Although u-PA, SK and performed plasminogen-SK complex have been therapeutically used in clinical studies on arterial occlusion in stroke as exogenous plasminogen activators, the systemic thrombolytic states are generated because circulating plasminogen and $\mathrm{Fbg}$ are markedly decreased or depleted. ${ }^{9)}$ Consequently, the risk of hemorrhage is augmented secondarily by possible lysis of fibrinstabilized hemostatic plugs which are protecting the sites of vascular disruption. Thrombolytic agents given systemically for treatment of stroke might produce intracerebral hemorrhage. However, in the present study, systemically applied t-PA produced extensive embolus dissolution 
without causing systemic plasminogen activation, Fbg breakdown or bleeding in SHRSPs.

In conclusion, clot-selective t-PA may be systemically applicable for the therapy of acute stroke as a thrombolytic agent which does not induce intracerebral hemorrhagic complications.

\section{References}

1) D. Collen, Thromb. Haemost., 43, 77 (1980)

2) D. C. Rijken and D. Collen, J. Biol. Chem., 256, 7035 (1981).

3) D. Pennica, W. E. Holmes and D. Collen, Nature (London), 301, 214 (1983).

4) R. J. Kaufman, L. C. Wasley and R. M. Kay, Mol. Cell. Biol., 5, 1750 (1985).

5) A. Hasegawa, H. Yamashita, S. Kondo, T. Kiyota, H. Hayashi, H. Yoshizaki, A. Murakami, M. Shiratsuchi and T. Mori, Biochem. Biophys. Res. Commun., 150, 1230 (1988).

6) M. Hoylaerts, D. C. Rijken, H. R. Lijnen and D. Collen, J. Biol. Chem., 257, 2912 (1982).

7) H. Kimata, K. Nakajima, S. Yamamoto and A. Hasegawa, $J$. Pharmacobio-Dyn., 13, 745 (1990).

8) A. P. Fletcher, N. Alkajarsig, M. Lewis, V. Tulevski, A. Davies, J. E. Brooks, W. B. Hardin, W. M. Landau and M. E. Raichle, Stroke, 7, 135 (1976).

9) G. J. del Zoppo, H. Zeumer and L. A. Harker, Stroke, 17, 595(1986).

10) E. Mori, M. Tabuchi, T. Yoshida and A. Yamadori, Stroke, 19, 802 (1988).

11) G. J. del Zoppo, A. Ferbert, S. Otis, H. Bruckmann, W. Hacke, J. Zyroff, L. A. Harker and H. Zeumer, Stroke, 19, 307 (1988).

12) H. Kimata, K. Nakajima, H. Suzuki, T. Koide and T. Narita, J. Pharmacobio-Dyn., 14, 399 (1991).

13) Y. Yamori, R. Horie, H. Handa, M. Sato and M. Fukase, Stroke, 7, 46 (1976).

14) D. C. Rijken, G. Wijngaards, M. Zaal-de Jong and J. Welbergen, Biochim. Biophys. Acta, 580, 140 (1979).
15) G. S. David and R. A. Reisfeld, Biochemistry, 13, 1014 (1974).

16) P. Friberger and M. Knos, "Chromagenic Peptide Substrates," ed. by M. S. Scully and V. V. Kakkar, Churchill Livingstone, Edinburgh, Scotland, 1979, p. 128.

17) “Hemorrhagic Diseases," 1st ed. by A. J. Quick, Lea and Febiger, Philadelphia, 1957, p. 379.

18) P. Coyle, Stroke, 17, 520 (1986).

19) M. Tagami, Y. Nara, A. Kubota, T. Sunaga, H. Maezawa, H. Fujino and Y. Yamori, Stroke, 18, 733 (1987).

20) O. Matsuo, D. C. Rijken and D. Collen, Nature (London), 291, 590 (1981).

21) C. Korninger, O. Matsuo, R. Suy, J. M. Stassen and D. Collen, J. Clin. Invest., 69, 573 (1982).

22) D. Collen, J. M. Stassen and M. Verstraete, J. Clin. Invest., 71, 368 (1983).

23) Ch. Mattsson, S. Nilsson and L. Haggroth, Thromb. Res., 30, 91 (1983).

24) E. J. Topol, A. A. Ciuffo, T. A. Pearson and B. H. Bulkley, J. Am. Coll. Cardiol., 5, 85 (1985).

25) S. R. Bergmann, K. A. A. Fox, M. M. Ter-Pogossian, B. E. Sobel and D. Collen, Science, 220, 1181 (1983).

26) F. Van de Werf, S. R. Bergmann, K. A. A. Fox, B. E. Sobel and D. Collen, Circulation, 69, 605 (1984).

27) W. Flameng, F. Van de Werf, J. Vanhaecke, M. Verstraete and D. Collen, J. Clin. Invest., 75, 84 (1985).

28) G. J. del Zoppo, B. R. Copeland, T. A. Waltz, J. Zyroff and L. A. Harker, Stroke, 17, 638 (1986)

29) H. H. Kaufman, J. H. Anderson, J. Woo, J. D. Huchton and D. C. Cannon, Acta Neurochir., 52, 185 (1980).

30) J. J. Emeis, C. M. Van den Hoogen and D. Jense, Thromb. Haemost., 54, 661 (1985).

31) C. Agnelli, M. R. Buchanan, F. Fernandez, J. Van Ryn and J. Hirsh, Blood, 66, 399 (1985).

32) H. Kimata, K. Nakajima, T. Koide, S. Yamamoto and S. Kondo, J. Pharmacobio-Dyn., 13, 751 (1990).

33) H. Kimata, T. Koide, K. Nakajima and S. Kondo, $J$ Pharmacobio-Dyn., 14, 25 (1991). 